\title{
Endoscopic removal of an impacted wooden toothpick in the wall of the sigmoid colon
}

\author{
Artur Zakościelny, Witold Zgodziński, Grzegorz Wallner, Krzysztof Zinkiewicz \\ $2^{\text {nd }}$ Department of General, Gastrointestinal Surgery and Surgical Oncology of the Alimentary Tract, Medical University of Lublin, \\ Lublin, Poland
}

Videosurgery Miniinv 2018; 13 (3): 417-419

DOI: https://doi.org/10.5114/wiitm.2018.75863

\begin{abstract}
Most ingested foreign bodies usually pass through the gastrointestinal tract without any complications. Sharp foreign bodies such as a wooden toothpick may cause severe complications, leading to an acute abdomen. They may also cause mild, non-specific gastrointestinal symptoms without significant findings. We describe a case of a 60-year-old man initially diagnosed with a foreign body impacted into the wall of the rectosigmoid junction upon screening colonoscopy. Incidentally, ingestion of the wooden toothpick 6 months before admission and the presence of recurrent fever and lower abdominal pain were confirmed in the patient's history. Our video case study demonstrates the successful endoscopic removal of the wooden toothpick impacted into the colon wall.
\end{abstract}

Key words: foreign body, screening colonoscopy, wooden toothpick, sigmoid colon.

\section{Introduction}

The majority of foreign bodies (FBs) pass through the entire gastrointestinal tract without complications [1, 2]. Approximately $10-20 \%$ of the cases require endoscopic removal and $\sim 1 \%$ need surgical intervention [3]. Rectosigmoid junction is one of the most common areas in which FBs may perforate the gut. The perforation of the sigmoid colon occurs in $<1 \%$ of cases $[4,5]$. In case of perforation of the colon laparoscopic colorectal surgery [6] or other modern endoscopic techniques, e.g. over-the-scope clip (OTSC) placement [7], should be considered.

We describe a case of a 60-year-old man diagnosed with a sharp FB impacted into the wall of the rectosigmoid junction upon screening colonoscopy, followed by successful endoscopic treatment.

\section{Case report}

A 60-year-old man was initially diagnosed with a FB (wooden toothpick) impacted into the wall of the sigmoid colon upon screening colonoscopy (Photos 1 A, B). Incidentally, ingestion of the wooden toothpick 6 months before admission and the presence of recurrent fever and lower abdominal pain were confirmed. Clinical examination revealed a white blood cell count of $52 \mathrm{~K} / \mu \mathrm{l}$ and an elevated C-reactive protein level of $25 \mathrm{mg} / \mathrm{ml}$. Abdominal X-ray and ultrasound were normal. Endoscopic removal of the FB was undertaken. The first attempt was performed under sedation but failed due to acute pain (Photo 2). The second attempt was performed in the operating theater under general anesthesia. A GIF-H180J videoscope (Olympus) was introduced into the sigmoid colon. Carbon dioxide $\left(\mathrm{CO}_{2}\right)$ was used for insufflation. Afterward, one side of the $\mathrm{FB}$, which was 

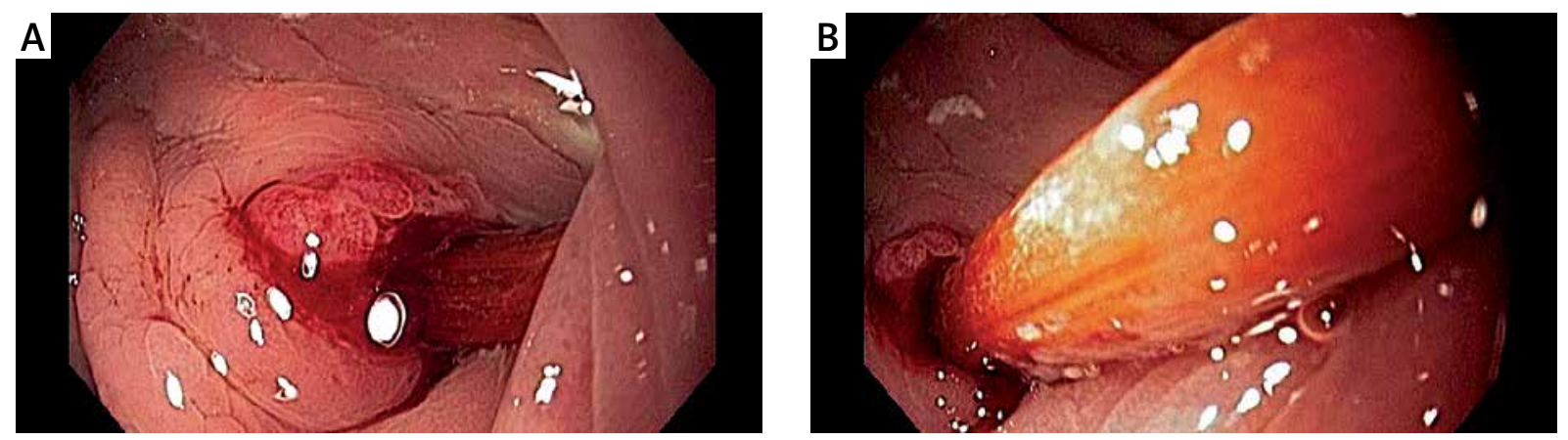

Photo 1. Endoscopic image of toothpick impacted in the colon wall

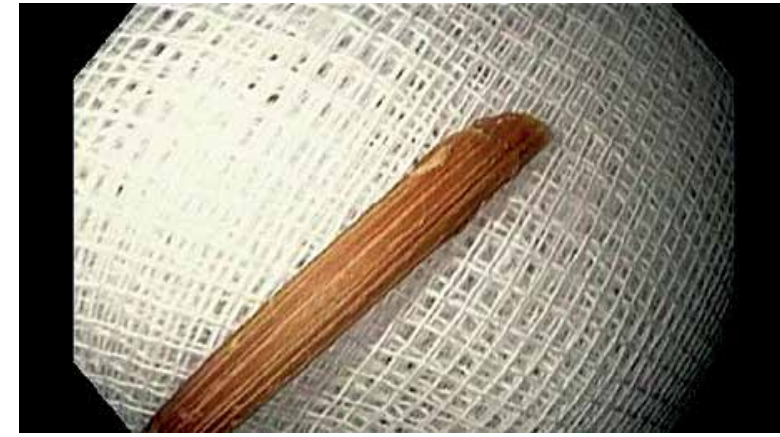

Photo 2. The wooden toothpick $(7 \mathrm{~cm} \times 0.3 \mathrm{~cm})$ after endoscopic removal from the recto-sigmoid junction

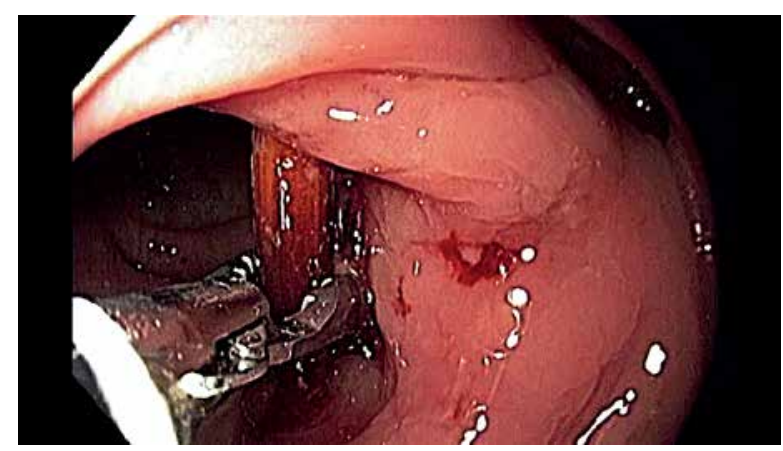

Photo 4. Endoscopic image of toothpick impacted in the colon wall

close to the wall of the intestine, was grasped with rat tooth grasping forceps, and it was removed after gentle pulling with the forceps and scope as shown in Video 1 (Photos 3, 4). The scope was reintroduced and small bleeding at the site of impaction was revealed. The perforation of the bowel was excluded by Veress needle puncture at Palmer's point. The patient did well clinically, having no evidence of active bleeding or perforation, and was discharged home on the fourth day after the procedure.

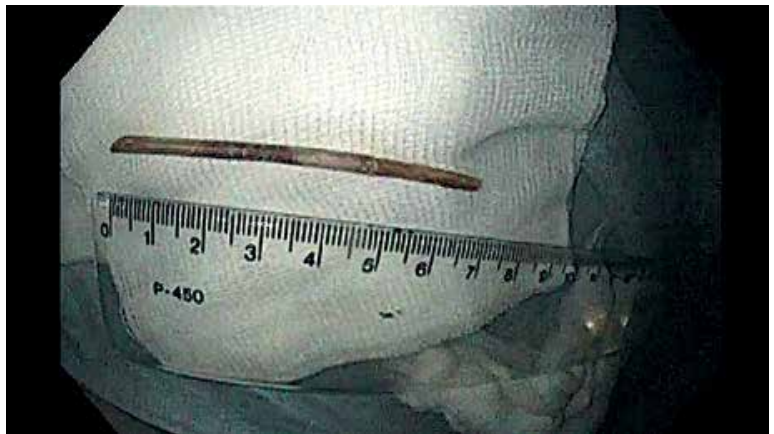

Photo 3. The wooden toothpick $(7 \mathrm{~cm} \times 0.3 \mathrm{~cm})$ after endoscopic removal from the recto-sigmoid junction

\section{Discussion}

Ingestion of foreign bodies (FB) is a common clinical problem. Especially sharp-ended, thin objects such as fish bones, metal objects and wooden toothpicks may cause life-threatening complications such as obstruction, Gl bleeding or gut perforation. The main risk factors associated with toothpick ingestion are a toothpick-chewing habit, rapid eating and mental disorders [8]. Selivanov reported that if the ingested foreign bodies reach the stomach, most of them (80\%) will pass uneventfully through the entire Gl tract and less than 1\% cause perforation [9]. Perforation happens more frequently in areas controlled by sphincters, physiological narrowing and acute flexures [10, 11]. The most commonly described locations include the duodenum, the caecum and the sigmoid colon. According to Budnick's 4-year survey performed in the United States from 1979 to 1982, 8176 reported "toothpick-related injuries" were found. The incidence rate of the ingested wooden toothpick was estimated at 3.6 per 100,000 persons per year. Five percent (0.2 per 100,000 per- 
sons per year) of these injuries involved internal organs such as mediastinal structures, the liver, bladder or even the aorta [10]. Correct diagnosis of an ingested wooden toothpick may be difficult. In the radiological images - abdominal X-ray, ultrasound or computed tomography - the presence of a toothpick can also be easily overlooked. Moreover, only $12 \%$ of patients were able to remember swallowing a toothpick [12]. The symptoms are mostly nonspecific, such as lower abdominal pain or recurrent fever, as in the present case report. According to $\mathrm{Li}$ and Ender, the onset of symptoms occurred from less than 1 day to 15 years after the event $[11,12]$. In our case study it was 6 months. Surgical treatment is mandatory in the presence of complications such as peritonitis, abscesses, fistulas or FBs migration to adjacent extra-colonic structures [3, 10, 12]. In the present case, we successfully identified and removed the impacted wooden toothpick during the second endoscopy. Perforation of the bowel was excluded during the procedure. The post-procedural course was uneventful and no complications were noted. This confirms that application of endoscopy to remove an impacted, sharp foreign body from the large bowel is safe and should be a first choice treatment in these difficult cases.

\section{Conclusions}

Our case study demonstrates the successful endoscopic removal of a wooden toothpick impacted into the colon wall. Endoscopy was confirmed to be the safest and most effective therapeutic approach for this difficult clinical problem.

\section{Conflict of interest}

The authors declare no conflict of interest.

\section{References}

1. Sarici IS, Topaz O, Sevim Y, et al. Endoscopic management of colonic perforation due to ingestion of a wooden toothpick. Am J Case Rep 2017; 18: 72-5.

2. Chung YS, Chung YW, Moon S, et al. Toothpick impaction with sigmoid colon pseudodiverticulum formation successfully treated with colonoscopy. World J Gastroenterol 2008; 14: 48-50.

3. Zezos P, Oikonomou A, Soutfas V. Endoscopic removal of a toothpick perforating the sigmoid colon and causing chronic abdominal pain: a case report. Cases J 2009; 2: 8469.

4. Luense S, Simon P, Heidecke CD, et al. Over-the-scope clip closure of a colon perforation caused by an ingested wooden toothpick. ACG Case Report J 2016; 3: e165.
5. da Mota FF, Lisbon TV, da Costa BX, et al. A novel clip-assisted method for endoscopic removal of an impacted toothpick from the colon. Endoscopy 2016; 48 Suppl 1: E259-60.

6. Dulskas A, Samalavicius NE, Gupta RK, et al. Laparoscopic colorectal surgery for colorectal polyps: single institution experience. Videosurgery Miniiv 2015; 10: 73-8.

7. Brodak M, Kosine J, Tacheci I, et al. Endoscopic treatment of a rectovesical fistula following radical prostatectomy by overthe-scope clip (OTSC). Videosurgery Miniinv 2015; 10: 486-90.

8. Lovece A, Asti E, Sironi A, et al. Toothpick ingestion complicated by cecal perforation: case report and literature review. World J Emerg Surg 2014; 9: 63.

9. Selivanov V, Sheldon GF, Cello JP, et al. Management of foreign body ingestion. Ann Surg 1984; 199: 187-91.

10. Budnick LD. Toothpick-related injuries in the United States, 1979 through 1982. JAMA 1984; 252: 796-7.

11. Chichom-Mefire A. Perforation of the splenic flexure of the colon by an ingested wooden toothpick. J Surg Case Rep 2015; 1: $1-2$.

12. Izumi J, Satoh K, Iwasaki W, et al. Small bowel obstruction caused by the ingestion of a wooden toothpick: the CT findings and a literature review. Intern Med 2017; 56: 657-60.

Received: 15.08.2017, accepted: 26.02.2018. 